This document is confidential and is proprietary to the American Chemical Society and its authors. Do not copy or disclose without written permission. If you have received this item in error, notify the sender and delete all copies.

\title{
Prediction of antibiotic interactions using descriptors derived from compound molecular structure
}

\begin{tabular}{|r|l|}
\hline Journal: & Journal of Medicinal Chemistry \\
\hline Manuscript ID & jm-2017-002049.R2 \\
\hline Manuscript Type: & Article \\
\hline Date Submitted by the Author: & O6-Apr-2017 \\
\hline Complete List of Authors: & $\begin{array}{l}\text { Mason, Daniel; University of Cambridge, Chemistry Department } \\
\text { Stott, Ian; Unilever R\&D Port Sunlight } \\
\text { Ashenden, Stephanie; University of Cambridge, Chemistry Department } \\
\text { Weinstein, Zohar; Boston University School of Medicine, } \\
\text { Karakoc, Idil; Sabanci Universitesi, Faculty of Engineering and Natural } \\
\text { Sciences } \\
\text { Meral, Selin; Sabanci Universitesi, Faculty of Engineering and Natural } \\
\text { Sciences } \\
\text { Kuru, Nurdan; Sabanci Universitesi, Faculty of Engineering and Natural } \\
\text { Sciences } \\
\text { Bender, Andreas; University of Cambridge, Chemistry Department } \\
\text { Cokol, Murat; Harvard Medical School }\end{array}$ \\
\hline
\end{tabular}

SCHOLARONE ${ }^{\mathrm{m}}$

Manuscripts 


\title{
Prediction of antibiotic interactions using descriptors
}

\section{derived from compound molecular structure}

\author{
Daniel J. Mason ${ }^{1}$, Ian Stott ${ }^{2}$, Stephanie Ashenden ${ }^{1}$, Zohar B. Weinstein ${ }^{3}$, \\ Idil Karakoc ${ }^{4}$, Selin Meral ${ }^{4}$, Nurdan Kuru ${ }^{4}$, Andreas Bender ${ }^{1,{ }^{*}}$, Murat Cokol ${ }^{4,5,6^{*}}$
}

1 Centre for Molecular Informatics, Department of Chemistry, University of Cambridge, Cambridge, UK

2 Unilever Research and Development, Port Sunlight, Wirral, UK

3 Boston University School of Medicine, Boston, Massachusetts, USA

4 Faculty of Engineering and Natural Sciences, Sabanci University, Tuzla, Istanbul, Turkey

5 Department of Molecular Biology and Microbiology, Tufts University School of Medicine;

6 Laboratory of Systems Pharmacology, Harvard Medical School, Boston, Massachusetts, USA

* to whom correspondence may be addressed

ab454@cam.ac.uk

murat_cokol@hms.harvard.edu

\begin{abstract}
Combination antibiotic therapies are clinically important in the fight against bacterial infections. However, the search-space of drug combinations is large, making the identification of effective
\end{abstract}


combinations a challenging task. Here, we present a computational framework that uses substructure profiles derived from drug molecular structures and predicts antibiotic interactions. Using a previously published data set of 153 drug pairs, we showed that substructure profiles are useful in predicting synergy. We experimentally measured the interaction of 123 new drug pairs, as a prospective validation set for our approach, and identified 37 new synergistic pairs. Of the 12 pairs predicted to be synergistic, 10 were experimentally validated, corresponding to a 2.8 -fold enrichment. Having thus validated our methodology, we produced a compendium of interaction predictions for all pairwise combinations among 100 antibiotics. Our methodology can make antibiotic interaction predictions for any antibiotic pair since it solely requires chemical structures as an input.

Keywords: Antibiotics, drug interactions, drug combinations, Escherichia coli, drug resistance, infectious diseases, combination therapy, machine learning, drug structure

\section{Introduction}

The rising prevalence of antimicrobial resistant microbes coupled with a dearth of novel antimicrobials underlies the importance of drug combinations in the fight against microbes ${ }^{1-3}$. However, when combined, drugs may have synergistic or antagonistic interactions, corresponding to higher or lower than expected efficacy, respectively ${ }^{4-6}$. Synergistic drug combinations have greater treatment potential against antimicrobial resistant microbes, due to increased efficacy versus single drug use and fewer side effects $^{1,7,8}$, in addition to introducing multiple barriers against resistance evolution ${ }^{7}$ This may be especially relevant for treatment of gram negative bacterial infections for which no new drug was admitted to the market in the last forty years ${ }^{4,9,10}$. 
The search for synergistic antibiotic combinations is hampered by the combinatorial magnitudes of even small compound libraries ${ }^{8,11}, 12$. The experimental testing of pairwise drug combinations against antimicrobial resistant microbes is a costly process that requires efficacy testing in many drug concentration combinations. Therefore, a comprehensive screen for discovering synergy is unfeasible in terms of resources and time. Combinatorial modeling is an in silico approach to prioritize drug pairs most likely to be synergistic ${ }^{9,10,13}$. Previous approaches have used drug physicochemical properties ${ }^{11,14}$ 15 and chemogenomics data ${ }^{13,16}$ to identify synergistic drug pairs, and drug targets and molecular fingerprints to predict adverse drug interactions ${ }^{14,15,17,18}$, or single agent dose responses across multiple cell lines ${ }^{19}$. The approaches that use computationally predicted properties of drugs are especially attractive since they can be used to make predictions on new drug pairs.

The consideration of substructures in chemical structures is a tenet in bioactivity modeling with applications ranging from bioavailability to potency. However, how chemical substructures may affect bioactivity of compound mixtures is poorly studied. In this study, we developed a machine learning workflow, CoSynE (Combination Synergy Estimation) that harnesses the substructure profiles of compounds to predict antibiotic interactions. CoSynE is initially trained with a recently published high resolution drug interaction dataset evaluating 153 pairs among 18 antibiotic compounds in $E$. coli ${ }^{8,16}$. The resulting models were used to predict synergistic and antagonistic interactions in a newly generated experimental validation set of 123 pairs. Finally, CoSynE was trained on all 276 combinations and used to predict interactions in all pairwise combinations of 100 antibiotics. Our results offer a systematic framework for a compound-centered understanding of drug interactions in microbial pathogens.

\section{Results}




\section{8x18 antibiotic interaction training set and antibiotic substructure profiles}

We used a recently generated antibiotic interaction screen involving 18 antibiotics, whose chemical structures are shown in Figure 1. These antibiotics covered a variety of mechanisms of action, with nine, four and four drugs targeting protein synthesis, DNA and cell wall, respectively (Table 1). All 153 pairwise combinations among 18 antibiotic pairs (training set) were assigned an interaction score (alpha) according to the Loewe additivity model for drug interactions, which assumes that a drug is additive with itself ${ }^{16-18}$. A negative, zero or positive score corresponds to synergy, additivity or antagonism, respectively ${ }^{8}$. The interaction scores of all the pairs in the training set are given in Supplementary Table 1 and represented as a heat map in Figure 2a left. We defined the interaction profile of a drug as all the alpha scores of a drug (each row or column of data in Figure 2a left). Drugs are clustered by their interaction profile similarity, defined as the rank correlation of two drug's interaction profiles. As expected, antibiotics with similar structures or mechanism of action, for example Gentamicin and Tobramycin (both aminoglycosides), are clustered together. The previous study reported 20 synergistic and 28 antagonistic antibiotic pairs among 153 pairs (13\% synergy and $18 \%$ antagonism), using threshold alpha $<-0.5$ for synergy and alpha $>1$ for antagonism. In this study, we used the same threshold for synergy and alpha $>0.5$ for antagonism. The clustering suggested that antibiotics that target protein synthesis are often synergistic. We found that antibiotic pairs where both drugs target protein synthesis is enriched for synergy, with 10 of the 36 pairs both involving drug that target protein synthesis showing synergy, corresponding to a 2.3-fold enrichment (Fisher's Exact Test, p-value: 1.1x10 $\left.{ }^{3}\right)$.

Mechanism of action based drug synergy prediction has been done in several studies. However, the determination of a drug's mechanism of action is a costly process. Here, we sought to use the structural features of antibiotics, which are freely available, to predict antibiotic synergy against gram negative 
bacteria. For each of these 18 antibiotics, we generated a substructure profile (SSP) based upon SMILES structural representation (Supplementary Table 2). Each antibiotic's SSP is a binary array corresponding to the existence or lack thereof of 166 MACCS structural keys (see Experimental Section) ${ }^{20}$. As a measure of structural similarity between two drugs, we calculated the Tanimoto similarity using their chemical structure, with ranges between 0 (no similarity) and 1 (perfect similarity). In Figure 2a right, structural similarity for all 153 drug pairs are shown. Drugs are clustered by correlation between structural similarity profiles. Consistent with their structural similarity, pairs CLA+ERY (both macrolides), CIP+LEV (both quinolones) and AMK+TOB (both aminoglycosides) are clustered together. This clustering is similar but not identical with the clustering done for alpha scores. To allow for comparison with the structural similarity or alpha score, we put glyphs inside each cell in Figure 2a. For example, CLA+ERY is structurally very similar (brown) and additive (white).

Figure 2a left and right are representations of two orthogonal observations (drug interaction and drug similarity) associated with a drug pair. A drug is additive with itself, hence the self-self combinations depicted in Figure 1 left are additive, with alpha score $=0$. A drug also has perfect similarity with itself, hence Figure 1 right depicts the structural similarity of self-self pairs as 1 . Therefore, drugs with high similarity will have interaction scores around 0 . Structural similarity and the absolute value of alpha had a weak negative correlation (Spearman's rank correlation $r=-0.16, p=4.8 \times 10^{-2}$ ) (Figure $2 b$ ). By definition, a drug has a perfect drug interaction profile similarity with itself. In agreement, structural similarity and drug interaction profile similarity were significantly positively correlated $(r=0.26, p=$ $1.1 \times 10^{-3}$ ) (Figure $2 \mathrm{c}$ ), as expected. The weak but significant correlation between structural similarity and alpha profile similarity, in addition with the suggestive and weak correlation of structural similarity and alpha supported that there is an association between chemical structure and drug interactions. Therefore, we next constructed a model to relate alpha scores and SSP features. 


\section{Using SSPs to predict antibiotic interactions}

Defining drugs as nodes and interactions as edges in a drug interaction network, an interaction score is an edge property, while SSP is a node property. In order to use the information depicted in two SSPs, we defined a jointSSP for a drug pair as the bit-wise average of two SSP's features (Figure 3). For each substructure, similarity is indicated with values 0 or 1 , while array values of 0.5 indicate substructures found in only one of the component drugs. We used the SSP of 18 drugs in the training set and generated joint SSP profiles for all 153 antibiotic pairs. Hence transforming the SSP of two drugs (two node properties) into a joint SSP (an edge property), we used machine learning classifiers to relate jointSSP and interaction type (synergy-additive-antagonism) of antibiotic pairs. Our machine learning workflow, CoSynE, outputs an interaction type prediction for an unseen drug pair using its jointSSP.

We used three different types of cross-validation (Figure 3; cross-validation types). (i) K-fold: $20 \%$ of the data was randomly selected and hidden from CoSynE in 10 randomly shuffled rounds. (ii) Leave drug out: All data for one drug was hidden from CoSynE. In both these cross-validation routines, the remaining data was used to train models that predict the hidden data. (iii) Leave pair out: All data for two drugs were hidden from CoSynE and synergy is predicted for the pair that is comprised of these two drugs. K-fold, leave drug out, or leave pair out cross validations correspond to the prediction of synergy for drug pairs where both drugs are known, one is unknown, or both are unknown to CoSynE, respectively. Therefore, performance across increasingly difficult scenarios for drug interaction prediction may be estimated. Although CoSynE makes predictions for any of these three types of drug interaction, we grouped additive and antagonistic together into one class (no synergy) while calculating our performance metrics. We explored 5 different classifier algorithms (Bernoulli Naïve Bayes, Decision Trees, k-Nearest Neighbours, Support Vector Machine, Random Forest, and Extremely Randomised 
Trees) (Supplementary Table 3) and since random forests had the best performance (Table 2), we used random forests in the rest of the study ${ }^{21}$. Table $2 \mathrm{a}$ real data rows show the performance metrics of random forests associated with each cross-validation method used, when real data is used as input to CoSynE (as opposed to node-shuffled or edge-shuffled data, described below). In K-fold cross validation, a strikingly good Matthews Correlation Coefficient (MCC) of 0.69 and area under ROC (AUROC) of 0.89 was obtained. Expectedly, these performances dropped with more difficult cross-validations. However, we observed an MCC score of 0.42 and AUROC of 0.78 even with the leave pair out cross-validation. These results strongly suggest that CoSynE produces reliable predictions for antibiotic interactions. However, they do not yet establish that SSPs are useful for drug interaction prediction, due to the following consideration.

It has been previously reported that drugs have a non-uniform tendency to show synergy, with promiscuously synergistic drugs serving as hubs in drug interaction networks ${ }^{2,3,8,22}$. For example, while the background synergy was $13 \%$ in our training set, pairs involving Fusidic Acid were synergistic in $53 \%$ of the cases. This raises the question if the high performance of CoSynE in K-fold and leave drug out models is due to over-fitting to the $18 \times 18$ drug interaction training data. Importantly, we note that the 'leave pair out' model is immune to this consideration, since no data is provided to CoSynE about either of the constituent drugs. In order to analyze this possible bias, we used two controls. (i) node-shuffled: we randomly shuffled the names and corresponding SSP for all drugs in the training set. (ii) edgeshuffled: we randomly shuffled the interaction outcome of each of the pairs. We note that the nodeshuffled control only randomly changes the names of drugs and leaves the drug pair interaction network's topology intact. This is expected to eliminate SSP related prediction power while leaving topology related prediction intact. However, the edge-shuffled control completely changes the topology of the network, which is expected to eliminate all predictive power. 
We tested CoSynE performance on node-shuffled and edge-shuffled data for each of the crossvalidation schemes described above. As expected, edge-shuffled data was not useful for prediction in all three types of cross-validation, with MCC scores around 0 and AUROC scores around 0.5 (i.e. equivalent to random selection). In the K-fold and leave drug out cross-validations, node-shuffled data resulted in better performance than edge-shuffled, with MCC scores $>0.2$ and AUROC $>0.8$. This indicates that some of the predictive power of CoSynE in these cross-validation schemes is due to synergy hubs. However, performance was consistently lower than the metrics obtained when the real drug interaction network was used for cross-validations, indicating that SSP is useful for the prediction of drug synergy. The predictive power of leave-pair-out cross-validation was completely abolished in node-shuffled data, with MCC and AUROC metrics similar to edge-shuffled data. Since the only difference between the real data and node-shuffled data is SSP of drugs, we therefore conclude that CoSynE successfully related drug synergy to chemical substructures as represented by SSPs.

\section{Experimental validation of predictions by CoSynE}

In order to assess the predictive power of CoSynE, we chose 6 additional drugs as a validation set (Kanamycin, Penicillin G, Roxithromycin, 5-fluorouracil, Mupirocin, and Pentamidine) (Figure 4a). Among these, KAN, PNG and ROX are "easy" drugs to predict for, since they are structurally similar to the antibiotics in the training set. For example, with a Tanimoto similarity of 0.78 , KAN is structurally very similar to TOB from the training set. From our observations in Figure $2 b$, it is expected that these antibiotics will have similar drug interaction profiles. In contrast, 5FU, MUP and PEN were "hard" drugs, since they were structurally different from the training set, with Tanimoto similarity below 0.15 to the drugs in the training set. In addition, 5FU is used as a cancer chemotherapy drug and PEN is used against protozoal infections, making these two drugs very different from all the rest of the drugs used in the 
study, which are all antibiotics. Importantly, none of these drugs have chemogenomic profiles reported in E. coli, thereby rendering it impossible to generate predictions using chemogenomics based methods. We generated the SSP for each of these drugs and predictions for all pairwise combinations of these 6 drugs and 18 antibiotics in the training set (108 pairs) and all pairwise combinations of 6 validation drugs (15 pairs). Validation pairs involving one training set compound and one validation set compound are "easy pairs" since CoSynE retains information about one of the constituent drugs. Conversely, validation pairs where both compounds are unknown to the model are "hard pairs". We note that easy or hard validation pairs are analogous to a leave compound out or leave pair out cross validation described above, respectively.

Next, we experimentally measured in duplicate the interaction of all pairs in the validation set, corresponding to 123 new drug interaction experiments (Figure 4b). We followed the exact same experimental setup as in the training $\operatorname{set}^{16}$ (see Experimental Section). Alpha scores of two experimental replicates had a very high correlation $\left(r=0.87, p=2.1 \times 10^{-39}\right)$, indicating the reliability of our drug interaction measures (Figure $4 \mathrm{~b}$ inset). We used the mean of two replicates as alpha score for each pair (Supplementary Table 1). The alpha profile of Kanamycin was correlated with the structurally similar drug Tobramycin used in training set $\left(r=0.71, p=1.5 \times 10^{-4}\right)$, as expected. Using the same thresholds as in the training set, we discovered a total of 37 new synergistic drug pairs.

We next compared CoSynE predictions with the validation set results (Table $2 \mathrm{~b}$ ). In the complete validation set, performance was at par with the CoSynE leave drug out cross-validation, with MCC 0.38 and AUROC 0.73. As expected, pairs with easy drugs received better performance values (MCC $=0.50$, $A U R O C=0.81)$ than pairs with hard drugs $(M C C=0.31, A U R O C=0.63)$. Also expected was that easy pairs received better performance values $(\mathrm{MCC}=0.38, \mathrm{AUROC}=0.74)$ than hard pairs $(\mathrm{MCC}=0.29$, $A U R O C=0.70)$. Importantly, even for hard pairs, which include two new drugs that were not part of the training set, performance was better than the node-shuffled data controls in Table 2a. The ROC and 
Precision-recall curves for all validation sets are shown in Figure $4 c$ and $4 d$, respectively. 10 of the 12 pairs predicted to be synergistic by CoSynE (with synergy probability higher than 0.5 ) were validated, corresponding to a 2.8 -fold increase in synergy among predictions (Fisher's exact test, $\mathrm{p}$-value: $10^{-4}$ ) (Figure 4e). This fold-increase is superior to the mechanism of action based synergy prediction power we observed above. More importantly, CoSynE can produce successful synergy predictions for drugs with no available mechanism of action, such as Pentamidine. For example, CoSynE successfully predicted PEN-FUS and PEN-ERY synergies, which are among easy pairs and hard pairs, respectively. In addition, the two strongest synergies that we experimentally found in our screen (PEN+TET and PEN+ROX) were correctly predicted to be synergistic by CoSynE. All synergistic drug pairs considered or reported in this study is given as a network in Figure 5.

Having thus validated our methodology, we next ranked all the substructures in terms of their importance in antibiotic interaction prediction after training on the full dataset of 24 compound combinations (Supplementary Table 4). We found that only 73 of the 166 substructures were used by CoSynE after the feature selection stage, and we counted the presence of each of these features in combinations that were synergistic, antagonistic, or additive (Supplementary Figure). Across each of the substructures, there is little to distinguish individual patterns that correspond to a mainly-synergistic combination. The most highly weighted feature \#19 (toward the left-hand side of Supplementary Figure)

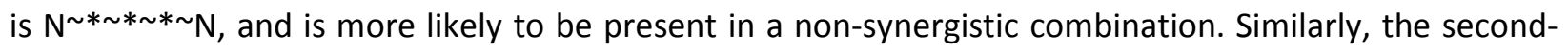
highest weighted feature \#29 is more associated with non-synergistic combinations, and is ${ }^{* \sim C H} \sim \mathrm{N}$. Features \#6, \#28, and \#17 are more likely to be associated with synergistic combinations, however these are not weighted as highly, and are substructures that involve double-bonded carbon. Examples of each of these examined substructures are highlighted on the 24 compounds used in the study. Our analyses of the substructure weights and frequency suggest that CoSynE interprets a complex combination of these features, rather than any single indicators, as predictors of synergy. 


\section{Large-scale prediction of antibiotic synergies using SSPs}

These observations verify that CoSynE can successfully predict interactions among drugs using only chemical structure derived SSPs. With the addition of the validation set to the training set, the entire drug interaction data considered in this study is a $24 \times 24$ matrix of drug pairs (Supplementary Table 1). The synergistic drug interactions learned from this screen is shown in Figure 5. To further strengthen our model, we trained CoSynE with all the data considered thus far (joint SSP and alpha scores for 276 drug pairs in E. coli). We used the K-fold, leave drug out and leave pair out cross-validations, as well as nodeshuffled and edge-shuffled data controls (Table 2c). The performance metrics and trends observed were similar to the cross-validation metrics for the training set. Especially important is that in the leave pair out cross-validation, CoSynE had an MCC of 0.29 and AUROC of 0.77 while the node-shuffled and edgeshuffled controls had MCCs around 0 and AUROC around 0.5 , indicating they have no predictive power. Since the only difference between the node-shuffled data and real data is the SSPs, we once again conclude that chemical structure derived SSPs are useful to predict antibiotic synergy. This trained model allows us to predict the pairwise interactions of any two compounds in E. coli with known chemical structure, subject to consideration of an appropriate applicability domain.

In silico model predictions are considered to be valid when a predicted entity falls within the applicability domain of a model. In other words, to be confident in our predictions of each new compound combination, we should know a threshold of distance over which performance is reduced to the point we cannot make a good prediction. We worked toward this goal by examining the continuous Tanimoto distance between each test combination and nearest neighbor in the training set during leavepair-out cross validation. Visual interpretation of MCC scores for test combinations relative to nearest neighbors in the training data suggested a general trend that confidence in a prediction nears random 
performance as a distance of 0.3 is approached for the $18 \times 18$ matrix, and as a distance of 0.4 is approached for $24 \times 24$ matrix.

We have thus established that we can generate reliable interaction predictions for antibiotic combinations in E. coli. CoSynE may therefore be used to produce a compendium of predicted antibiotic synergy among a large number of antibiotics. To this end, we generated a list of 100 antibiotics and produced their associated SSPs. The antibiotic list included the 24 compounds used in this study, while the rest of the antibiotics were either with known chemogenomic profile or included in recent largescale antibiotic resistance studies ${ }^{6,8}, 23,24$. Using CoSynE and the SSP of these 100 antibiotics, we produced a 100x100 matrix of predictions, covering 4950 pairs in total (Supplementary Table 5). Among these pairs, 691 were predicted to be synergistic, and 215 of those were within a conservative distance threshold of 0.15 ; our estimated performance based upon leave-pair-out cross validation at this continuous Tanimoto distance is a precision of approximately $60 \%$. We therefore expect around 400 of these novel combinations to exhibit synergistic interaction against $E$. coli.

\section{Discussion and Conclusions}

In this study, we developed and experimentally validated a computational framework, CoSynE, that can successfully predict synergistic antibiotic pairs using only descriptors derived from chemical structure of constituent drugs. Since chemical structures are readily available for most compounds of interest in this regard, our methodology can make predictions for any pairwise drug interaction for a given phenotype. 
This is in contrast with prediction methods that require experimental data on each considered drug. As we demonstrated with the enriched synergy among protein synthesis inhibitors, mechanism of action is also a predictor of synergy. However, mechanism of action information is not available for many drugs and that which is available may not be of relevance in the organism of interest. For example, Pentamidine's poorly understood mechanism of action in bacteria limits making mechanism of action based predictions for Pentamidine, whereas CoSynE can make predictions based on the structure of Pentamidine alone.

Chemogenomic or microarray data sets, when available, may be used as a substitute for the mechanism of action of a drug, enabling objective snapshots of a drug's effect on a phenotype. These studies have allowed a genome-wide analysis of drug mechanism of action, and have been successfully incorporated into pairwise drug synergy prediction algorithms. A particularly strong aspect of these studies is that they allow observations on the genome-wide effects of a synergistic drug combination. However, a weakness is that in order to make a prediction for a new drug, one needs new experimental work resulting in chemogenomic or microarray data sets for the drug of interest. The same weakness holds when the MIC of drugs is used for drug synergy prediction. Since CoSynE uses only structural information, once the training dataset has been screened no experimental effort is required to obtain descriptors for the novel compounds that make up future predictions. This offers an advantage over approaches such as that by Kaitlyn et $a .^{19}$, which required single agent dose information for 40 compounds across 27 melanoma cell lines to train a random forest model that predicted mutant BRAF melanoma anti-tumour combinations, and would require the same dose information to make predictions for new compounds. While Kaitlyn et al. achieve an AUROC of 0.87 and accuracy of 0.82 during 10 -fold cross-validation, this is lower than our 5-fold cross-validation for the $18 \times 18$ training data $(A \cup R O C=0.89$, accuracy $=0.93)$ and $24 \times 24$ model $(A \cup R O C=0.89$, accuracy $=0.86)$ that requires only the knowledge of chemical structures to make novel predictions. Experimental validation by Kaitlyn et 
al. shows that their model achieves a higher recall and slightly higher precision compared to our overall performance for easy and hard validation data, though the accuracy of CoSynE is slightly higher at 0.76 compared to that of Kaitlyn et al. of 0.64 .

Another drawback of using experimental drug related data for predicting drug interaction is the noise associated with experimental data. Especially with large-scale screens, data reproducibility is often far from perfect, weakening any inference using these data. By limiting our analysis to only chemical structures and synergy, we circumvent the need for experimental data to predict synergy for a pair. In addition, chemical structures are always known, hence the substructure profiles of compounds are known with perfect precision and at no cost, and only require standardization with regard to their representation. Using CoSynE, we produced a compendium of predictions for all pairwise interactions among 100 compounds. Our analysis allowed a ranking of substructures by their importance in antibiotic interaction prediction. Of the 166 substructures analyzed in this study, 73 were selected by CoSynE for synergy predictions. Our analysis identified substructures that are most predictive of drug interactions. A disadvantage of CoSynE over prediction methods that use biological information about the organism or test system used in the assay is that the predictions can only be made for the same species that the drug interaction data has been obtained for in the first place.

The experimental drug interaction data used in this study, all pairwise interactions among 24 compounds, is one of the largest drug interaction data sets in a bacterial species. This is partially made possible by the use of a miniaturized checkerboard assay, which is reproducible and efficient, and we experimentally discovered 37 new synergistic compound pairs using this approach. The sensitivity and specificity of CoSynE will improve simply with more drug interaction data, since chemical structures of compounds are already known. However, the quality of the data sets and broad sampling of chemical and activity space is also important. In this study, we were able to achieve a 2.8 -fold enrichment for synergy. This level of enrichment is very significant in practical terms. For example, consider that to fully 
screen 100 compounds in pairs corresponds to 4,950 combinations, and that this makes for a total of 178,200 data points (assuming a $6 \times 6$ dose-response matrix). In such cases, a $64 \%$ reduction in the total number of measurements required is a very attractive prospect in terms of both time and cost.

Since we can make prediction for any drug pair, as we have done for 100 drugs, the next challenge is improving these predictions. Our recommendation would be to gather more (good quality) combinations that gradually expand the chemical and biological space represented by the available chemical libraries ${ }^{25}$. In an industrial setting, this approach may be iterative, following a pipeline of training-prediction-screening-training of novel combinations. The results from each round incorporated into CoSynE will make more informed predictions, fueling the discovery for novel synergistic combinations.

\section{Experimental Section}

\section{Generation of combination descriptors and feature selection}

Structural compound fingerprints were calculated using RDKit $\mathrm{v} 2015^{20}$, representing each compound as 166 MACCS structural keys, and machine learning models were constructed using Scikit-Learn v0.17 ${ }^{26,26}$ and Python v2.7. Owing to differences in cross validation performance observed during development of our tool, MACCS keys were chosen over a hashed Morgan fingerprint representation (2048 bits, radius 2). Given the small size of our dataset relative to the number of features generated, it was necessary to reduce the number of features used to train each model, per prediction type, and per descriptor set. This was achieved for classification tasks by ranking all features in the training data per descriptor set according to their ANOVA F-value. With this information, $99 \mathrm{SVM}^{27}$ classifiers were tested using the 1 st to 100th percentile of features (Radial Basis Function kernel, $C=0.1$, gamma $=0.001$ ). Each percentile was 
subjected to 10 repeats of stratified and shuffled 5-fold cross validation in order to determine which percentile of ranked features provides the best performance (highest scoring Matthews Correlation Coefficient, MCC) for prediction of synergistic combinations. In each case, the training of feature selectors utilized only data available at the time of training to avoid information leak from the test set; the same as throughout this study. At the end of this stage, the optimal percentile of ranked features to use per descriptor set were stored for use in future model construction.

\section{Classifier performance metrics}

Since this is a classification problem, we must use classification performance metrics that consider whether the prediction made was synergistic (positive) or non-synergistic (negative), and whether this is in fact true or false. The counts of these outcomes, also together with the strength of the prediction can be used to calculate the Area Under the Receiver Operating Curve (AU-ROC), which tells us the trade-off between the true positive and false positive prediction rate. For the AU-ROC value, 0.5 corresponds to random performance, a value of 1 to a perfect model, and values above approximately 0.7 are conventionally accepted as a 'satisfactory' model (though this always depends on the dataset at hand). The Matthews Correlation Coefficient (MCC) is another classification metric that considers all possible outcomes of a classification problem, whereby a value of 0 corresponds to a random model, 1 to a perfect model, and values above approximately 0.5 correspond to a 'satisfactory' model (though again, this is highly dataset-dependent). In our analyses we made use of MCC as the metric to optimize for, since this considers all possible outcomes of a classification prediction. We use this in preference to accuracy, which only considers the correct proportion of true positive and true negative predictions that have been correctly classified; if $10 \%$ of the dataset is positive, but everything has been predicted negative, the model performance would be very low, but the accuracy is still $90 \%$. We included performance in terms of accuracy and other metrics in Table 2. 


\section{Parameter searching and model choice}

To select the best-performing classification approach per descriptor set, each estimator (k-nearest neighbors; KNN, random forest; RF, extremely randomized trees; ET, support vector machine; SVM, decision tree; DT, Bernoulli naïve Bayes; BNB) was subjected to a grid search for parameters that maximize the objective function of MCC in a stratified and shuffled 5-K-Fold. Upon finding the best set of parameters for this scenario, the model was further subjected to 10 repeats of stratified and shuffled 5K-Folds, and the average MCC across all repeats was stored. The best model-descriptor configurations were selected based upon the highest average MCC.

\section{Cross-validation of selected models}

Models that were selected as best-performers based upon the K-fold stage were subjected to two further cross-validation scenarios to gain further insight into predictive capability; leave-drug-out and leave-pair-out cross-validation. The leave-drug-out test is repeated for as many compounds as there are in the training data (i.e. 18 folds for the $18 \times 18$, before disregarding any folds without synergistic pairs) and the average performance per fold is calculated. In the leave-pair-out scenario, where both compounds are unknown to the model, this test is repeated for as many combinations there are in the training data (i.e. 153 folds), and statistics are calculated across each held-out pair at the end.

\section{Applicability domain analysis}

The applicability domain of a model provides an indication of how reliable predictions are as a function of distance from the training data. With a final model trained upon all available compound combination data, we performed the leave-pair-out cross-validation, and kept a record of the continuous Tanimoto distance (1-Tc) between the held-out combination and the remainder of the training data together with how correct the prediction was. This enabled us to plot performance metrics versus binned ( 5 linear bins between 0 and 1) distances for 276 splits in a difficult prediction scenario. 


\section{Drug interaction experiments}

Escherichia coli MG1655 strain was used for all experiments. The minimal inhibitory concentration for each drug tested is provided in Table 1. All drugs were purchased from Sigma at a purity >=95\%. Drug interactions were evaluated based on growth inhibition in a simplified $4 \times 4$ checkerboard assay, using tryptic soy buffer media. In this experimental setup, antibiotics are linearly titrated on each axis ranging from zero to minimal inhibitory concentration. 552 files corresponding to all pairwise interaction experiments in duplicate among 24 drugs considered in this study are given as Supplementary Data. 256 of these experiments were newly conducted in this study. Each file has 16 rows corresponding to 16 conditions in a $4 \times 4$ matrix of drug concentration combinations. In each row, $\mathrm{OD}_{600}$ readings of an $E$. coli culture in a drug condition is given every 15 minutes for 12 hours, measured in a Tecan Infinity F200 microplate reader. For each drug pair $\mathrm{X}$ and $\mathrm{Y}$, drugs in filename are ordered alphabetically; small $\mathrm{a} / \mathrm{b}$ corresponds to two replicates. 16 rows correspond to the following conditions: 1) $X 0+Y 0,2) X 0+Y 1,3)$ $X 0+Y 2,4) X 0+Y 3,5) X(+Y 0,6) X 1+Y 1,7) X(+Y 2,8) X(+Y 3,9) X 2+Y 0,10) X 2+Y 1,11) X 2+Y 2,12) X 2+Y 3,13)$ $X 3+Y 0,14) X 3+Y 1,15) X 3+Y 2,16) X 3+Y 3$. The growth phenotype was approximated by the area under the optical density curve over 12 hours. The interaction score for each $4 \times 4$ growth matrix was determined using the isobologram analysis described in reference 8. Matlab codes that use experimental data to score pairwise drug interaction scores (alpha) are provided as Supplementary Data.

Supporting Information includes Supplementary Figure, Supplementary Tables 1-5 and Supplementary Data.

\section{Conflict of Interest}


The authors declare that they have no conflict of interest.

\author{
Acknowledgements \\ This work was supported by a grant from Unilever Research and Development to DJM, NIGMS Training \\ Program in Biomolecular Pharmacology T32GM008541 to ZBW, Turkish Academy of Sciences GEBIP \\ Programme and TUBITAK 1155934 Grant to MC, and an ERC Starting Grant (MIXTURE) to AB.
}

\title{
Corresponding Author Information
}

Andreas Bender:

email: ab454@cam.ac.uk

phone: +44 1223762983

Murat Cokol:

email: murat cokol@hms.harvard.edu

phone: +1 6174326164

\begin{abstract}
Abbreviations used
5FU, 5-fluorouracil; AMK, amikacin; AUROC, area under receiver operating curve; BNB, Bernoulli naïve Bayes; CEF, cefoxitin; CHL, chloramphenicol; CIP, ciprofloxacin; CLA, clarithromycin; CoSynE, Combination Synergy Estimation; DT, decision tree; ERY, erythromycin; ET, extremely randomized trees; F1, harmonic mean of the precision and recall score; FUS, fusidic acid; GEN, gentamycin; KAN, kanamycin; KNN, k-nearest neighbors; LEV, levofloxacin; MACCS, Molecular ACCess System; MCC, Matthews Correlation Coefficient; MIC, minimal inhibitory concentration; MUP, mupirocin; NAL,
\end{abstract}


nalidixic acid; NIT, nitrofurantoin; OXA, oxacillin; PEN, pentamidine; PNG, penicillin G; PR, precision recall; RF, random forest; RIF, rifampicin; ROC, receiver operating curve; ROX, roxithromycin; SMILES, simplified molecular-input line-entry system; SPE, spectinomycin; SSP, substructure profile; SVM, support vector machine; TET, tetracycline; TOB, tobramycin; TRI, trimethoprim; VAN, vancomycin.

\section{References}

1. Lehar, J.; Krueger, A. S.; Avery, W.; Heilbut, A. M.; Johansen, L. M.; Price, E. R.; Rickles, R. J.; Short, G. F.,3rd; Staunton, J. E.; Jin, X.; Lee, M. S.; Zimmermann, G. R.; Borisy, A. A. Synergistic drug combinations tend to improve therapeutically relevant selectivity. Nat. Biotechnol. 2009, 27, 659-666.

2. Alanis, A. J. Resistance to antibiotics: are we in the post-antibiotic era? Arch. Med. Res. 2005, 36, 697705.

3. Levy, S. B.; Marshall, B. Antibacterial resistance worldwide: causes, challenges and responses. Nat. Med. 2004, 10, S122-129.

4. Brown, D. Antibiotic resistance breakers: can repurposed drugs fill the antibiotic discovery void? Nat. Rev. Drug Discov. 2015, 14, 821-832.

5. Cokol, M. Drugs and their interactions. Curr. Drug Discov. Technol. 2013, 10, 106-113.

6. Zimmermann, G. R.; Lehar, J.; Keith, C. T. Multi-target therapeutics: when the whole is greater than the sum of the parts. Drug Discov. Today 2007, 12, 34-42.

7. Yeh, P. J.; Hegreness, M. J.; Aiden, A. P.; Kishony, R. Drug interactions and the evolution of antibiotic resistance. Nat. Rev. Microbiol. 2009, 7, 460-466.

8. Cokol, M.; Chua, H. N.; Tasan, M.; Mutlu, B.; Weinstein, Z. B.; Suzuki, Y.; Nergiz, M. E.; Costanzo, M.; Baryshnikova, A.; Giaever, G.; Nislow, C.; Myers, C. L.; Andrews, B. J.; Boone, C.; Roth, F. P. 
Systematic exploration of synergistic drug pairs. Mol. Syst. Biol. 2011, 7, 544.

9. Bulusu, K. C.; Guha, R.; Mason, D. J.; Lewis, R. P.; Muratov, E.; Motamedi, Y. K.; Cokol, M.; Bender, A. Modelling of compound combination effects and applications to efficacy and toxicity: state-ofthe-art, challenges and perspectives. Drug Discov. Today 2016, 21, 225-238.

10. Spitzer, M.; Griffiths, E.; Blakely, K. M.; Wildenhain, J.; Ejim, L.; Rossi, L.; De Pascale, G.; Curak, J.; Brown, E.; Tyers, M.; Wright, G. D. Cross-species discovery of syncretic drug combinations that potentiate the antifungal fluconazole. Mol. Syst. Biol. 2011, 7, 499.

11. Yilancioglu, K.; Weinstein, Z. B.; Meydan, C.; Akhmetov, A.; Toprak, I.; Durmaz, A.; lossifov, I.; Kazan, H.; Roth, F. P.; Cokol, M. Target-independent prediction of drug synergies using only drug lipophilicity. J. Chem. Inf. Model. 2014, 54, 2286-2293.

12. Borisy, A. A.; Elliott, P. J.; Hurst, N. W.; Lee, M. S.; Lehar, J.; Price, E. R.; Serbedzija, G.; Zimmermann, G. R.; Foley, M. A.; Stockwell, B. R.; Keith, C. T. Systematic discovery of multicomponent therapeutics. Proc. Natl. Acad. Sci. U. S. A. 2003, 100, 7977-7982.

13. Jansen, G.; Lee, A. Y.; Epp, E.; Fredette, A.; Surprenant, J.; Harcus, D.; Scott, M.; Tan, E.; Nishimura, T.; Whiteway, M.; Hallett, M.; Thomas, D. Y. Chemogenomic profiling predicts antifungal synergies. Mol. Syst. Biol. 2009, 5, 338.

14. Gottlieb, A.; Stein, G. Y.; Oron, Y.; Ruppin, E.; Sharan, R. INDI: a computational framework for inferring drug interactions and their associated recommendations. Mol. Syst. Biol. 2012, 8, 592.

15. Yamanishi, Y.; Pauwels, E.; Kotera, M. Drug side-effect prediction based on the integration of chemical and biological spaces. J. Chem. Inf. Model. 2012, 52, 3284-3292.

16. Chandrasekaran, S.; Cokol-Cakmak, M.; Sahin, N.; Yilancioglu, K.; Kazan, H.; Collins, J. J.; Cokol, M. Chemogenomics and orthology-based design of antibiotic combination therapies. Mol. Syst. Biol. 2016, 12, 872.

17. Loewe, S. Die quantitativen probleme der pharmakologie. Ergeb. Physiol. 1928, 27, 47-187. 
18. Greco, W. R.; Bravo, G.; Parsons, J. C. The search for synergy: a critical review from a response surface perspective. Pharmacol. Rev. 1995, 47, 331-385.

19. Gayvert, K. M.; Aly, O.; Platt, J.; Bosenberg, M. W.; Stern, D. F.; Elemento, O. A Computational approach for identifying synergistic drug combinations. PLoS Comput. Biol. 2017, 13, e1005308.

20. Landrum, G. Rdkit.org. Accessed March 13, 2017.

21. Breiman, L. Random Forests. Mach. Learning 2001, 45, 5.

22. Farha, M. A.; Brown, E. D. Chemical probes of Escherichia coli uncovered through chemical-chemical interaction profiling with compounds of known biological activity. Chem. Biol. 2010, 17, 852862.

23. Imamovic, L.; Sommer, M. O. Use of collateral sensitivity networks to design drug cycling protocols that avoid resistance development. Sci. Transl. Med. 2013, 5, 204 ra132.

24. Oz, T.; Guvenek, A.; Yildiz, S.; Karaboga, E.; Tamer, Y. T.; Mumcuyan, N.; Ozan, V. B.; Senturk, G. H.; Cokol, M.; Yeh, P.; Toprak, E. Strength of selection pressure is an important parameter contributing to the complexity of antibiotic resistance evolution. Mol. Biol. Evol. 2014, 31, $2387-2401$.

25. Paricharak, S.; IJzerman, A. P.; Bender, A.; Nigsch, F. Analysis of iterative screening with stepwise compound selection based on Novartis in-house HTS data. ACS Chem. Biol. 2016, 11, 12551264.

26. Pedregosa, F.; Varoquaux, G.; Gramfort, A.; Michel, V.; Thirion, B.; Grisel, O.; Blondel, M.; Prettenhofer, P.; Weiss, R.; Dubourg, V. Scikit-learn: Machine learning in Python. J. Mach. Learn. Res. 2011, 12, 2825-2830.

27. Cortes, C.; Vapnik, V. Support-vector networks. Mach. Learning 1995, 20, 273-297. 


\section{Figure Legends:}

Figure 1: Structures and target processes for 18 antibiotics used in the training set. Abbreviations are defined in Table 1 and given in the Abbreviations List.

Figure 2: $18 \times 18$ antibiotic interaction training set and antibiotic substructure profiles. (a) Left: Drug interaction scores for each pair of 18 antibiotic compounds used as training data in the study. Synergistic, additive or antagonistic pairs are shown with green, white or red, respectively. Compounds are clustered by their alpha score profile similarity. Note that the cells on the diagonal are white, since a drug is additive with itself. The glyphs inside each cell corresponds to the structural similarity between two drugs, as indicated by the colorbar on the right. Right: Tanimoto similarity for each pair of the 18 antibiotic compounds in the training set. Structurally different or similar compound pairs are shown with white or red, respectively. Compounds are clustered by structural similarity. Note that the cells on the diagonal are red, since a drug has perfect similarity with itself. The glyphs inside each cell correspond to the drug interaction between two drugs, as indicated by the colorbar on the left. (b) Scatter plot for absolute value of alpha vs. structural similarity for each pair, represented by blue circles. A weak yet significant negative correlation supports that structurally similar compounds have alpha scores close to 0 (Spearman $r=-0.16, p=4.8 \times 10^{-2}$ ). (c) Scatter plot for alpha profile similarity vs. structural similarity for each pair, represented by blue circles. A weak yet significant positive correlation supports that structurally similar compounds have similar drug interaction profiles (Spearman $r=0.26, p=1.1 \times 10^{-3}$ ).

Figure 3: Schematic of the workflow to use SSPs to predict antibiotic interactions. For training input, CoSynE uses two data sets: (i) A set of substructural keys per compound, which are based upon the SMILES notation of each compound. For every compound pair, we use the average of the SSPs of the compounds, generating a jointSSP. (ii) Prior experimentally screened compound combination data. Features are analyzed as a whole, and filtered for those which are most representative of the dataset. 
The classification performance of several methods using different settings (hyperparameters) is then tested via three cross-validation routines to obtain the best-performing CoSynE model. For validation, we generate the jointSSP for novel combinations by the same descriptors, reducing to the same informative features, and querying the model with this data to yield a predicted synergy type and an estimated probability of this being the case. Predictions by CoSynE are experimentally tested and analyzed for prospective validation performance.

Figure 4: Experimental validation of predictions by CoSynE. (a) Structures for six compounds used in validation set. (b) In the heatmap, the interaction scores for all pairs in validation experiments for CoSynE predictions are shown. Left and right side of heatmap are experimental results for duplicates. Colormap is identical to Figure 1 left, hence synergistic, additive or antagonistic pairs are shown with green, white or red, respectively. Drugs are arranged by easy/hard drugs (blue/magenta names) on the $x$ axis, with vertical blue/magenta line separating pairs with easy or hard drugs. Drugs are arranged by easy/hard pairs (black/red names) on the y axis, horizontal black/red line separating easy or hard pairs. The inset shows the agreement between the interaction scores of two duplicate experiments $(r=0.87, p$ $\left.=2.1 \times 10^{-39}\right)$. Pairs with easy or hard drugs are shown in blue or magenta circles, respectively. Hard pairs are shown with red asterisks. (c, d) ROC and PR curves for the validation sets. (d) Scatter plot for the probability of synergy as predicted by CoSynE and experimentally obtained alpha scores.

Figure 5: Network representation of all drug synergies considered or reported in this study. 
Table 1: Name, abbreviation, pubchem id, minimum inhibitory concentration, and target process of all 24 drugs used in this study. Validation set drugs are shown in the bottom, with blue and magenta representing easy and hard drugs, respectively.

Table 2: Performance metrics of CoSynE using random forests. In a and c, three different crossvalidation results with real data, node-shuffled data or edge-shuffled data are given for training set or final model, respectively. In b, performance metrics for validation set are given for complete data, pairs with easy/hard drugs or easy/hard pairs. 


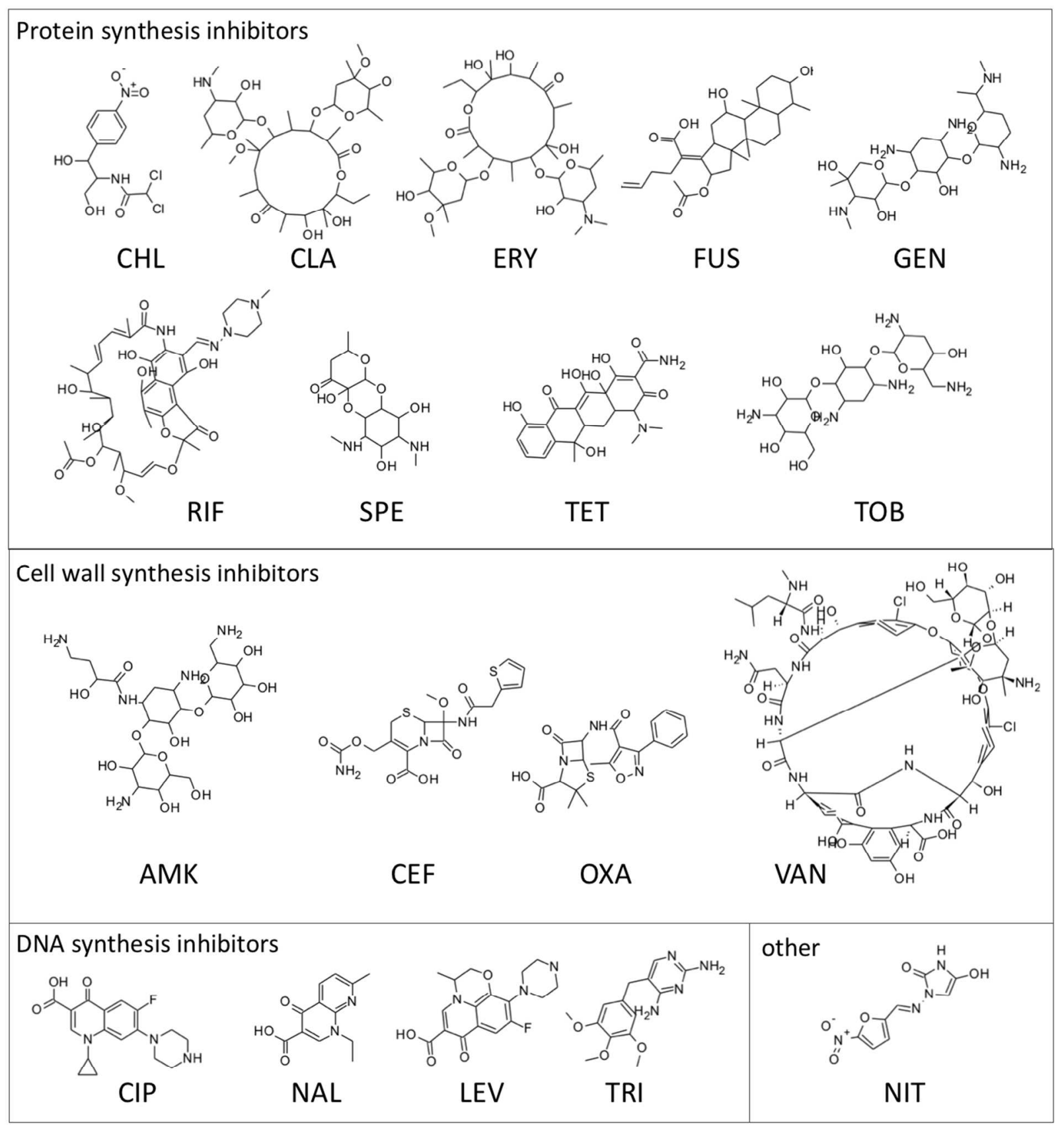

Figure 1. 


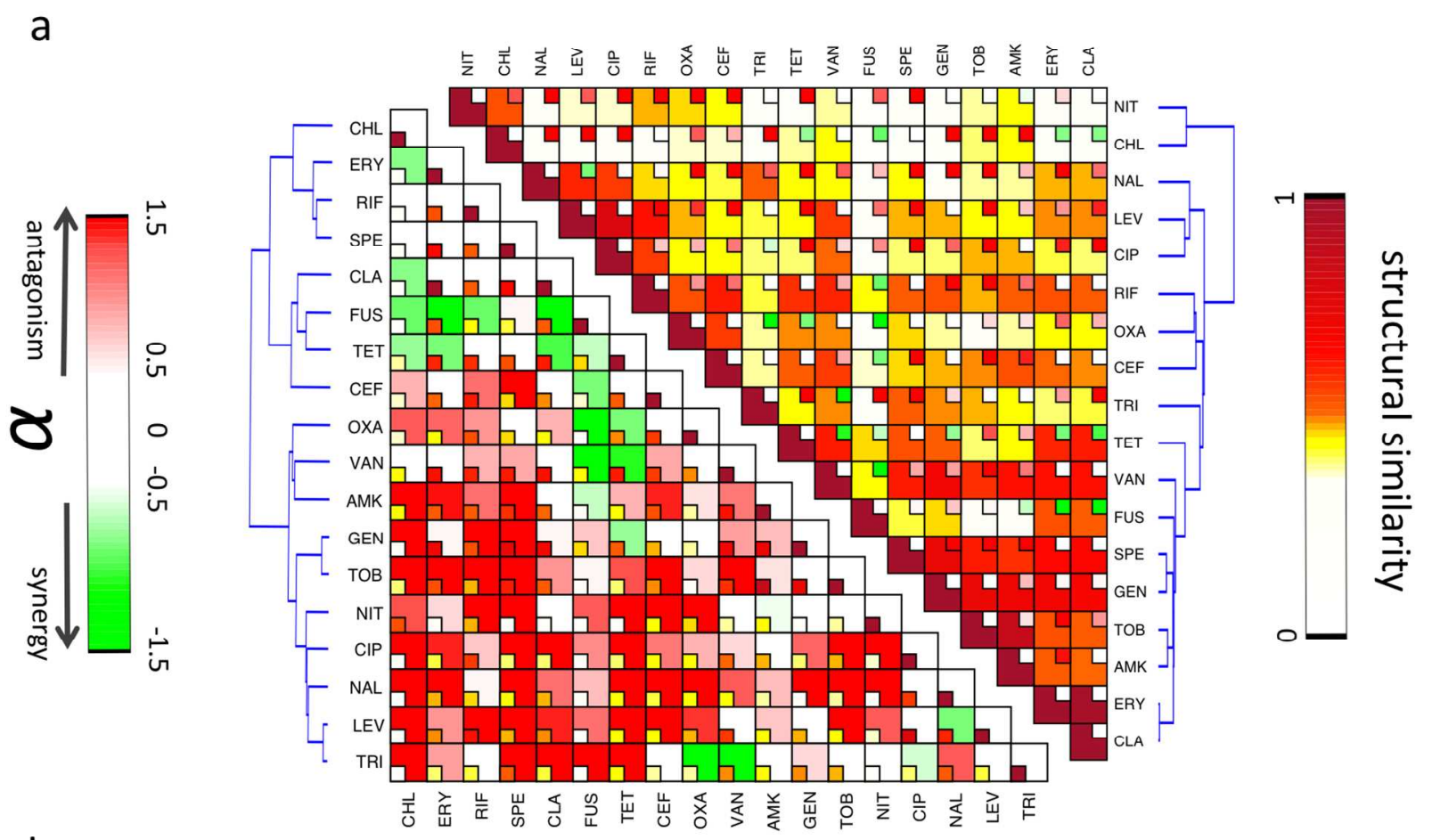

b

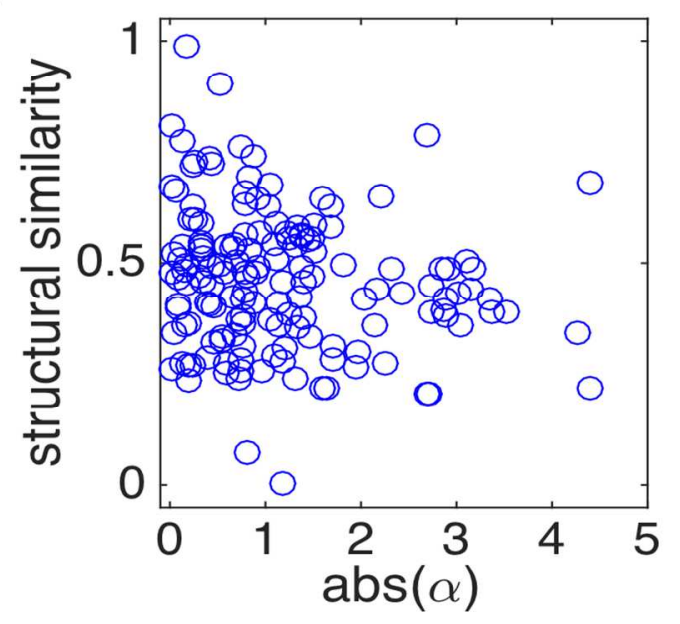

C

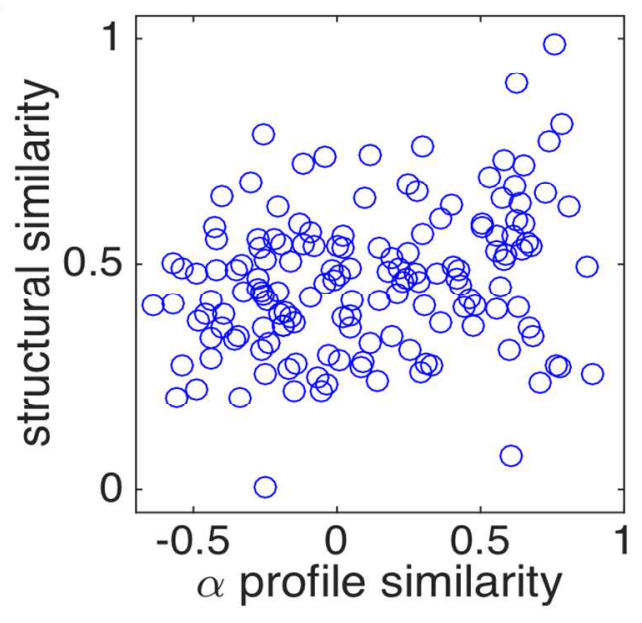

Figure 2. 


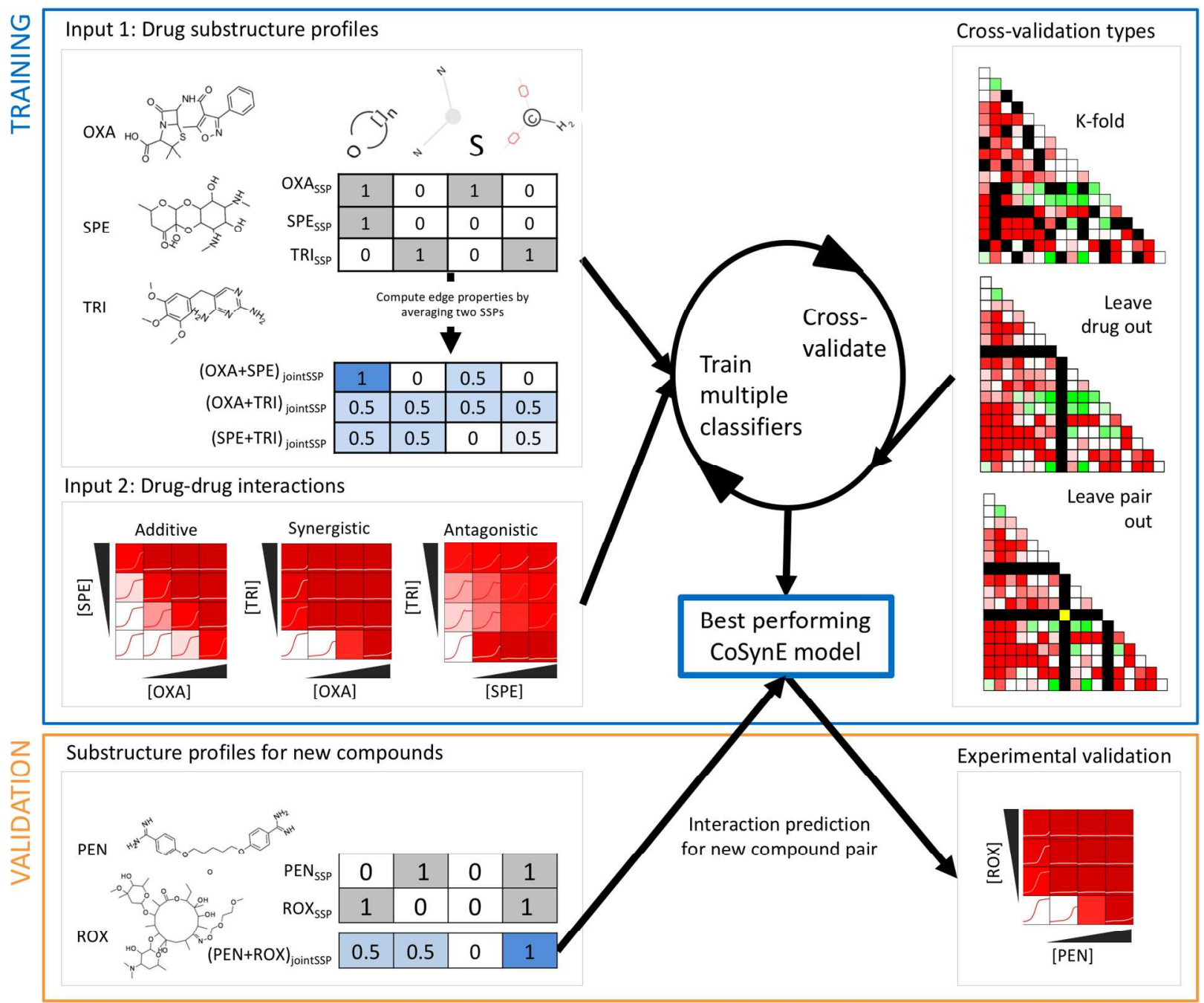

Figure 3. 
a

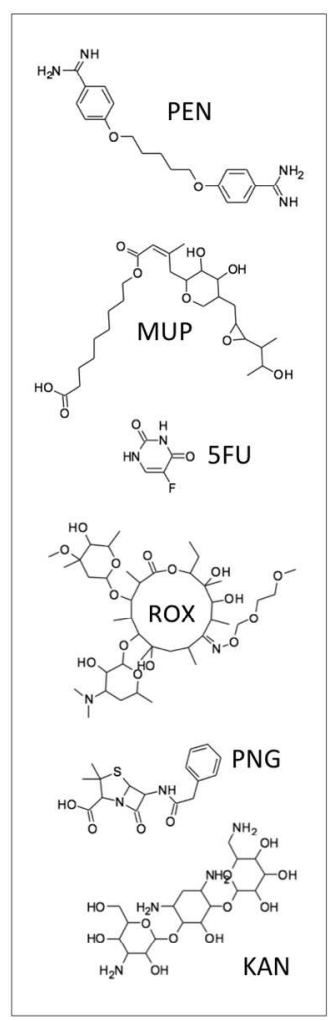

b

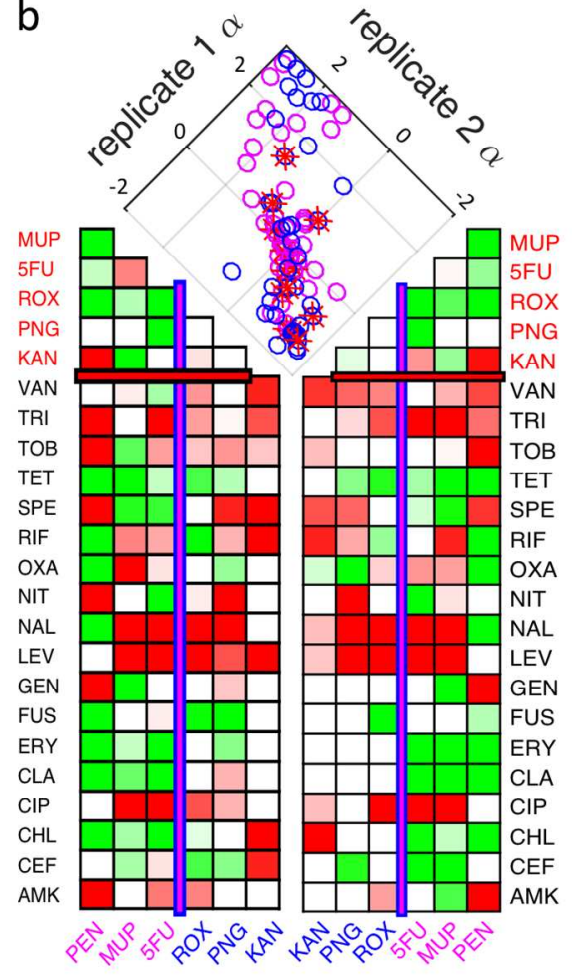

C

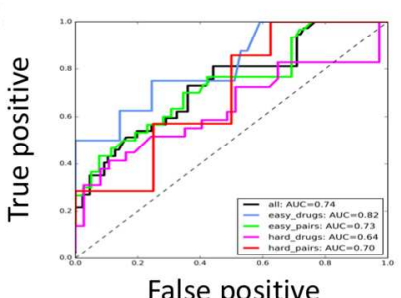

d

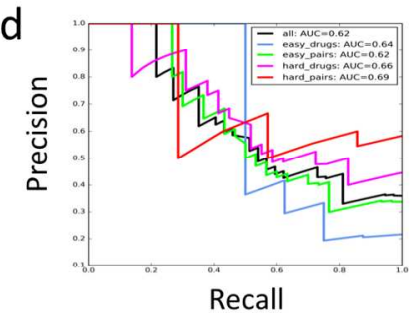

e

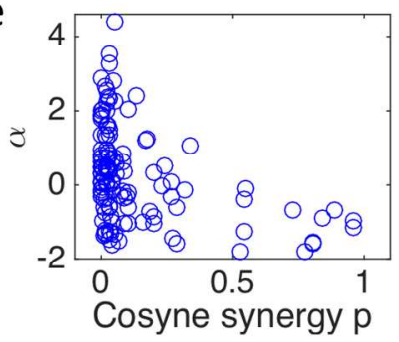

Figure 4. 


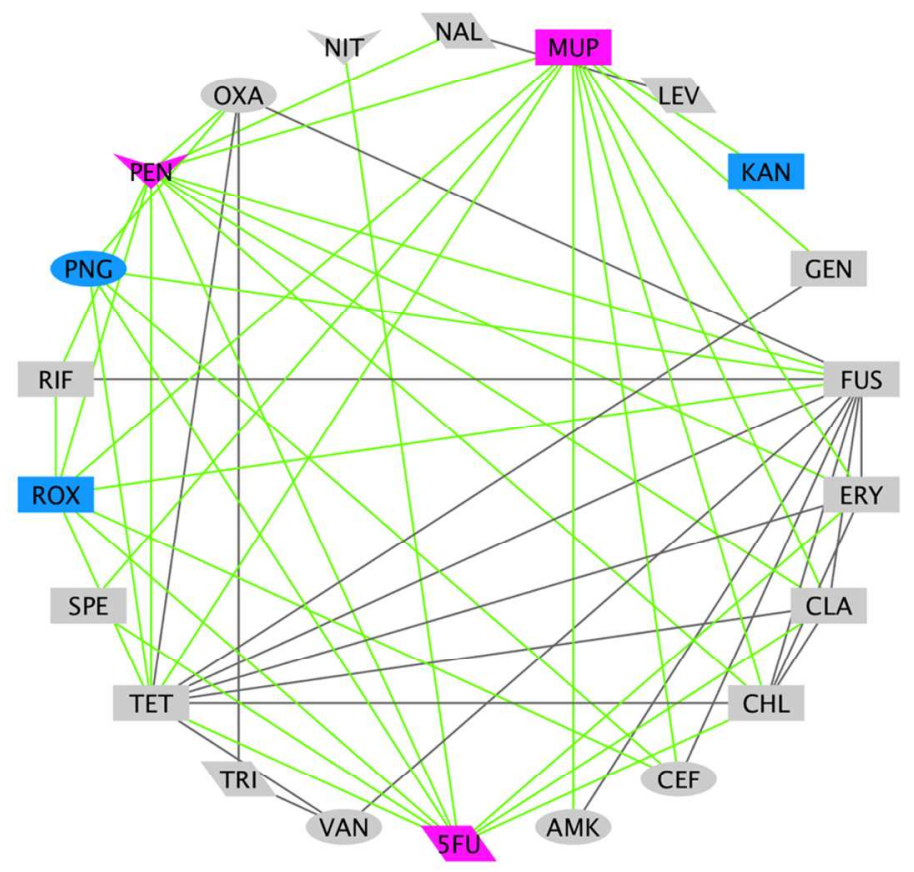

\begin{tabular}{|l|}
\hline Drug type \\
$\square$ Hard drugs \\
$\square$ Easy drugs \\
$\square$ old drugs \\
Synergy type \\
$\square$ Novel \\
$\square$ Previously reported \\
Drug target process \\
$\square$ DNA synthesis \\
$\square$ Cell wall synthesis \\
$\bigcirc$ Protein synthesis \\
$\square$ Other
\end{tabular}

\section{Figure 5.}




\begin{tabular}{|c|c|c|c|c|c|c|}
\hline & & Drugname & Abbreviation & pubchemid & Target process & MIC (ug/ml) \\
\hline \multirow{18}{*}{\multicolumn{2}{|c|}{ 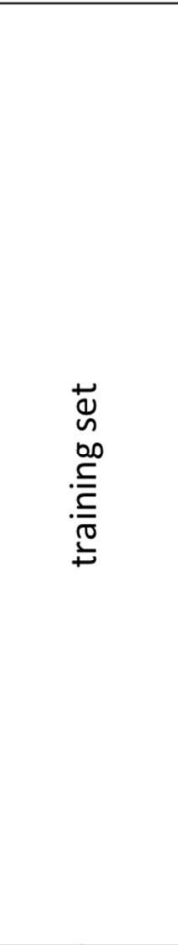 }} & Amikacin & AMK & 37768 & cell wall synthesis & 4 \\
\hline & & Cefoxitin & CEF & 441199 & cell wall synthesis & 4,5 \\
\hline & & Chloramphenicol & $\mathrm{CHL}$ & 5959 & protein synthesis & 5 \\
\hline & & Ciprofloxacin & CIP & 2764 & DNA synthesis & 0,01 \\
\hline & & Clarithromycin & CLA & 84029 & protein synthesis & 22 \\
\hline & & Erythromycin & ERY & 12560 & protein synthesis & 13 \\
\hline & & Fusidic acid & FUS & 3000226 & protein synthesis & 800 \\
\hline & & Gentamicin & GEN & 3467 & protein synthesis & 3 \\
\hline & & Levofloxacin & LEV & 149096 & DNA synthesis & 0,013 \\
\hline & & Nalidixic acid & NAL & 4421 & DNA synthesis & 5 \\
\hline & & Nitrofurantoin & NIT & 6604200 & other & 12 \\
\hline & & Oxacillin & OXA & 6196 & cell wall synthesis & 190 \\
\hline & & Rifampicin & RIF & 5381226 & protein synthesis & 2,5 \\
\hline & & Spectinomycin & SPE & 15541 & protein synthesis & 5,6 \\
\hline & & Tetracycline & TET & 54675776 & protein synthesis & 18 \\
\hline & & Tobramycin & TOB & 36294 & protein synthesis & 2,5 \\
\hline & & Trimethoprim & TRI & 5578 & DNA synthesis & 0,35 \\
\hline & & Vancomycin & VAN & 21733102 & cell wall synthesis & 180 \\
\hline \multirow{6}{*}{ 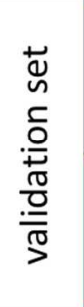 } & \multirow{3}{*}{ đิ } & Kanamycin & KAN & 6032 & protein synthesis & 14 \\
\hline & & Penicillin G & PNG & 5904 & cell wall synthesis & 20 \\
\hline & & Roxithromycin & ROX & 5480431 & protein synthesis & 62 \\
\hline & \multirow{3}{*}{ 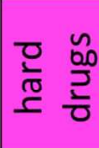 } & 5-Fluorouracil & $5 \mathrm{FU}$ & 3385 & DNA synthesis & 25 \\
\hline & & Mupirocin & MUP & 446596 & protein synthesis & 66 \\
\hline & & Pentamidine & PEN & 4735 & other & 100 \\
\hline
\end{tabular}

Table 1. 


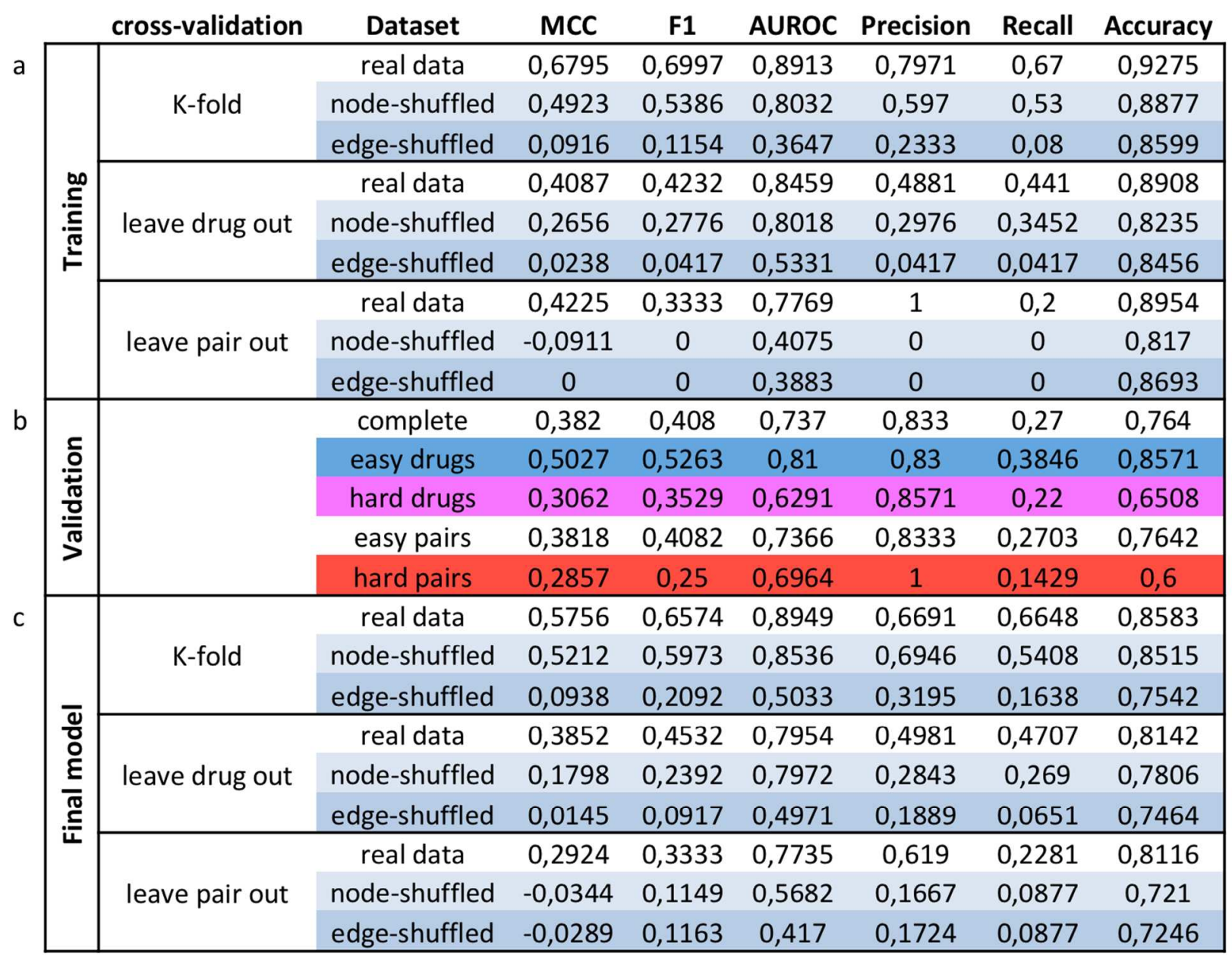

Table 2. 


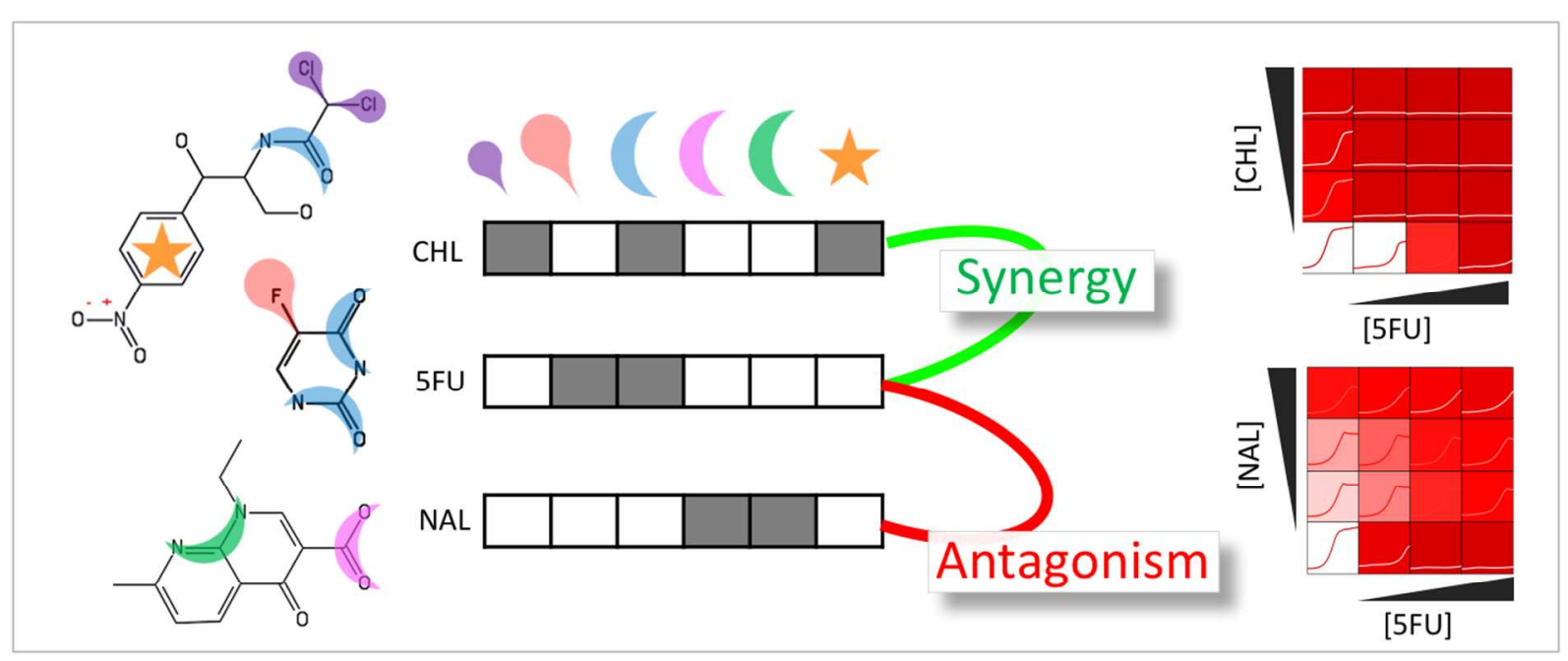

Table of contents Image

2

3

5

6

7

10

11

12

13

14

15
16

17

18

19

20

21

23

24

25

26

27

28

29

30

31

32

33

34

35

36

37

38

39

40

41

42

43

44

45

46

47

48

49

50

51

52

53

54

55

56

57

58

59

60 